\title{
GENERACIÓN DE VIAJES DE AEROPUERTOS EN LA CIUDAD DE GUAYAQUIL, ECUADOR
} GENERATION OF AIRPORT TRIPS IN THE CITY OF GUAYAQUIL, ECUADOR

\section{FEDERICO G. VON BUCHWALD DE JANON', CYNTHIA VALAREZO RAMÓN² , CAROLINA GÓMEZ MALDONADO ${ }^{3}$}

\footnotetext{
1 Universidad Católica de Santiago de Guayaquil. fritzvb25@icloud.com

2 Universidad Católica de Santiago de Guayaquil.

3 Departamento Técnico de la empresa Consulaudi Cia. Ltda.
}

RESUMEN

Se determinó la demanda de tráfico que se genera en el aeropuerto de Guayaquil (José Joaquín de Olmedo) usando como material de apoyo el manual de generación de viajes realizado por el ITE (Institute of Transportation Engineers) de Estados Unidos. En el manual se establecen tres variables independientes para este uso de suelo, las que son: empleados que laboran en el aeropuerto de Guayaquil, vuelos totales durante el día y vuelos comerciales (nacional e internacional). Además se consideró el flujo vehicular a la entrada y a salida del aeropuerto. Se realizaron conteos de usuarios a pie, personas dentro de los vehículos durante la hora pico en 3 días y conteos únicamente de vehículos en 24 horas. Se obtuvo información de la cantidad de personas que laboran en el aeropuerto, los vuelos que se generan durante el día y los vuelos comerciales. Con los datos obtenidos se determinaron las tasas de generación de viajes, coeficientes de correlación, ecuaciones de regresión y comparaciones de las gráficas obtenidas de Trip Generation del aeropuerto con las establecidas por el Manual de generación de viajes ITE (EEUU).

PALABRAS CLAVE: generación de viajes, coeficiente de correlación, ecuaciones de regresión.

\section{ABSTRACT}

The traffic demand generated in the Guayaquil's airport (José Joaquín de Olmedo) was determined using The Trip Generation Manual made by the Institute of Transportation Engineers (ITE) in the United States. Three independent variables were considered in this study: employees working at Guayaquil airport, total flights during the day and commercial flights (national and international) and vehicular flow in the entrance and exit of the airport was also determined.

The number of users was collected counting the pedestrians and persons in cars during the peak time in the 3 days and the vehicles during 24 hours. The information of the number of people working at the airport, flights generated during the day and commercial flights, was used to determine the trip generation rates, correlation coefficients, regression equations and the comparisons with the graphs established by the ITE Trip Generation Manual (USA).

KEYWORDS: trip generation, correlation coefficients, regression equations. 
INTRODUCCIÓN

Las diferentes actividades económicas demandan movilidad de las personas, éstas se deben identificar para posteriormente establecer qué tipo de infraestructura urbana se debe implementar en las diferentes zonas. Cuando estas actividades se concentran en un lugar se convierten en polos generadores de viajes. El objetivo de este estudio es determinar el volumen de tránsito que genera la operación del aeropuerto José Joaquín de Olmedo.

El progresivo congestionamiento en las vías de la ciudad de Guayaquil genera impactos viales, motivo por el cual se requiere investigar qué los genera. Para estos estudios se toman en cuenta las características y variables que son causantes de los problemas de accesibilidad vial y seguridad de un establecimiento. En razón de no tener estudios de generación de viajes del aeropuerto José Joaquín de Olmedo y aunque los resultados no se ajustan necesariamente a las condiciones de Guayaquil por sus diferentes circunstancias socio-económicas, se toman los datos presentados en las tablas del "Trip Generation Manual" (TCM) del "Institute of Transportation Engineers” (ITE).

Se realizaron conteos manuales a la entrada y salida de vehículos y conteos de ingreso de personas en las distintas puertas externas del aeropuerto. Con los valores obtenidos por medio del formato presentado por el "Trip Generation Manual" del ITE, se obtuvieron las correlaciones entre el número de viajes y las variables independientes, la desviación estándar, ecuación de regresión, gráficos y tablas para la demanda vehicular y el gráfico de número de viajes por unidad de tiempo. La finalidad del análisis de este estudio es ofrecer a las entidades interesadas una ecuación de tendencia de generación de viajes como aporte para la implementación futura de aeropuertos en Guayaquil.

\section{ANTECEDENTES} los registro de la Dirección General de Aviación
Civil en ese año en el aeropuerto se registraron 543,542 salidas y 528,415 entradas.

\section{JUSTIFICACIÓN}

Dado que en Guayaquil no existe un libro de referencia como lo tiene Estados Unidos (Trip Generation Manual del ITE), ni se tiene estudios relacionados que sirvan como parámetro para determinar el número de vehículos que generan los aeropuertos, se justifica esta investigación con el único aeropuerto que tiene la ciudad.

\section{OBJETIVO}

Determinar las tasas de generación de viajes en el aeropuerto de Guayaquil en función de las variables independientes: número de empleados, número de vuelos totales y número de vuelos comerciales por día.

\section{MATERIAL Y MÉTODO}

Además del uso del manual Trip Generation "User's Guide and Handbook", 9na edición, se revisarán varios archivos de tesis y trabajos de maestrías (en páginas web) sobre la determinación de tasas de generación de viajes.

\section{BASES PARA UNA GENERACIÓN DE VIAJES}

El Institute of Transportation Engineers por medio de su libro "User's Guide and Handbook estipula bases fundamentales para la realización y estimación de la generación de viajes; para el análisis de un nuevo estudio se deben argumentar los resultados del mismo por medio de la información emitida por el trip generation. Se conciertan diferentes metodologías necesarias para el análisis:

Que el tipo de uso de suelo que será analizado se encuentre según la codificación en el libro "Trip Generation Manual".

Determinar los volúmenes generados de viajes durante los días de estudio, para la definición de la hora pico de la misma.

Por medio de tasas de generación obtener las gráficas que se presentan en el Trip Generation Manual, en éstas se establece la relación de número de viajes que se originan en el uso de suelo estudiado versus variable independiente.

Obtención de la ecuación de regresión, la misma que relaciona la variable independiente con el número de viajes generados para cada día de estudio.

Descripción del Gráfico “Trip Generation”

Tasa de viajes promedio: es el promedio ponderado del número de viajes por unidad de variable independiente, este promedio se obtiene 
de la sumatoria de todos los viajes divididos para la suma de todas las variables independientes (Institute of Transportation Engineers, 2012).

Desviación estándar: es una medida de dispersión de los puntos alrededor de su media, a menor dispersión, la desviación estándar es menor. Gráficamente, el uso de la media ponderada de la tasa asume una relación lineal pasando a través del origen con la misma inclinación de la tasa. (Girardotti, 2001).

Para el estudio realizado las estadísticas son justificadas en porcentajes ponderados y no en porcentajes matemáticos, por ende, la desviación estándar es solo una aproximación y no es estadísticamente correcta (Institute of Transportation Engineers, 2012).

Análisis de Regresión: El manual define como coeficiente de determinación el porcentaje de variación del número de viajes relacionado con la variación del tamaño de la variable independiente. Por consiguiente si $\mathrm{R}^{2}$ es 0,75 , se refiere al $75 \%$ de la variación del número de viajes es representado por la variación del tamaño de la variable independiente. Mientras el valor de $\mathrm{R}^{2}$ más se aproxima a 1,0, la curva es más representativa, por lo que $\mathrm{R}^{2}$ debe ser mayor o igual a 0,5 .

USO DE SUELO SELECCIONADO PARA ESTUDIO

Se clasifica los aeropuertos de forma comercial y general, el aeropuerto de Guayaquil maneja ambos tipos, por ende para el análisis de generación de viajes en el número de vuelos se consideraron los vuelos totales incluyendo los de carga y los comerciales.

\section{DESCRIPCIÓN DEL LUGAR DE ESTUDIO}

El aeropuerto se encuentra ubicado al norte de Guayaquil, tiene 1480 empleados, estacionamiento para 1100 vehículos. El estudio abarca las cuatro puertas de acceso externas en donde una es de ingreso de vehículos, dos son para salida de vehículos y una de entrada/salida de usuarios a pie con anexo a la estación del sistema de transporte público (Metrovia).

\section{SELECCIÓN DE LAS VARIABLES INDEPENDIENTES}

El ITE (Institute of Transportation Engineers, 2012) muestra en su manual de Trip Generation tres variables que se deben considerar para un estudio de generación de viajes en aeropuertos:

- Número de empleados

- Número de vuelos totales en el día

- Número de vuelos comerciales en el día
Para vuelos totales se consideran todos los vuelos que registra el aeropuerto, incluyendo los cancelados.

\section{METOdología PARA EL LEVANTAMIENTO DE LA INFORMACIÓN}

En campo se levantaron los siguientes datos:

- Conteo de vehículos

- Conteo de usuarios

- Conteo de personas dentro de vehículos

- Encuestas a los usuarios

Con las autorizaciones pertinentes por parte de la operadora del aeropuerto (TAGSA) se planificó un estudio que abarca conteos durante 3 semanas, los que se consignaron en un formato con las descripciones correspondientes:

- Primera semana: conteo de vehículos durante 72 horas (3 días x 24 horas).

- Segunda semana: conteo de usuarios que pasaron por las 2 puertas de ingreso, desde las 6:45 hasta las 20:00.

- Tercera semana: conteo de personas dentro de los vehículos que entraban y salían del aeropuerto durante las horas pico de cada día de estudio. Para ello se estableció un formato considerando los tipos de vehículos, en entrada y salida, en intervalos de $15 \mathrm{~min}$.

Los datos se recopilaron por medio de un contador múltiple, sin considerar el tráfico de calles adyacentes al uso de suelo a ser estudiado.

Los resultados de los conteos sirvieron para definir las variables:

- Variable independiente: la información dada por el operador TAGSA.

- Variable dependiente: La hora pico generada según el conteo obtenido de vehículos.

Con esta información se obtuvo:

- Volúmenes generados durante el día

- Tablas de tasas de generación de viajes, que son de uso comparativos con las tasas generadas por el Trip Generation de aeropuertos de Estados Unidos.

- Ecuación de regresión.

Para ajustar el conteo de vehículos de los días seleccionados con el volumen anual se hizo una 
relación con la información de los volúmenes de pasajeros que registra el Sistema de Transporte Masivo de Guayaquil METROVIA durante un año para determinar que la fecha en la que se hicieron los conteos corresponden o no al promedio de los volúmenes diarios en un año y determinar en cuanto incide el promedio (en más o en menos). El análisis se hizo en cada uno de los días en los que se realizaron conteos y se comparó con el promedio de pasajeros del mismo en un día laborable (lunes a viernes) en el año de estudio, el cual resultó ser de 437.135 pasajeros y, se obtuvo que los días de conteos representan un 107\% (promedio ponderado de los días de conteos) del día promedio. Con esta condición se han realizado ajustes en los volúmenes de viajes a un $7 \%$ menos y se consideraron los días laborables como referencia.

\section{RESULTADOS Y DISCUSIÓN OBTENCIÓN DE TASAS DE GENERACIÓN DE VIAJES}

La estimación de tasa hace uso de los volúmenes generados durante la jornada AM y PM, tal como lo expresan las tablas del Trip Generation Manual.

La avenida próxima al aeropuerto que co- $^{-}$ munica a los sectores del centro con el norte de la ciudad es muy congestionada, por lo que un porcentaje significativo de vehículos ingresan al aeropuerto, usando esta vía como vía de paso, lo que distorsiona la demanda real de pasajeros que genera el aeropuerto, es por esto que se descontó este volumen adicional (14\% para la mañana en ambos días y $22 \%$ y $18 \%$ en la tarde para martes y miércoles).
TABLA 1. TASAS DE GENERACIÓN DE VIAJES PROMEDIO DÍA LABORABLE

\begin{tabular}{|c|c|c|c|c|}
\hline 总 & $\begin{array}{l}\text { VARIABLES } \\
\text { INDEPENDIENTES }\end{array}$ & $\begin{array}{r}\text { TASA } \\
\text { PROMEDIO } \\
\text { PONDERADA* }\end{array}$ & $\begin{array}{r}\text { RANGO DE } \\
\text { TASAS* }\end{array}$ & $\begin{array}{r}\text { RANGO } \\
\text { DE TASAS } \\
\text { AJUSTADAS } \\
\text { A UN 7\% } \\
\text { MENOS }\end{array}$ \\
\hline \multirow{3}{*}{ am } & Empleados & 0,9 & $0,8-0,93$ & $0,7-0,87$ \\
\hline & Promedio de vuelos por día & 10,7 & $10,03-11,67$ & $9,0-10,85$ \\
\hline & Vuelos comerciales por día & 12,0 & $11,27-13,24$ & $9,8-12,31$ \\
\hline \multirow{3}{*}{$\mathrm{pm}$} & Empleados & 0,9 & $0,76-1,00$ & $0,8-0,93$ \\
\hline & Promedio de vuelos por día & 11,1 & $9,51-12,54$ & $10,5-11,67$ \\
\hline & Vuelos comerciales por día & 12.4 & $10,68-13,70$ & $11,9-12,75$ \\
\hline
\end{tabular}

\section{ANÁLISIS DE REGRESIÓN Y GRÁFICAS (ILUSTRACIONES) DE GENERACIÓN DE VIAJES}

Al haber sólo un aeropuerto en la ciudad de Guayaquil, no es posible mostrar más puntos y crear con éstos una recta que permita definir una ecuación de generación de viajes, por lo que el análisis que se muestra a continuación es únicamente una comparación entre el punto obtenido con los datos locales de Guayaquil y el valor para cada una de las variables independientes analizadas en la hora pico obtenido en la jornada am y pm en un día laborable en la ecuación de generación de viajes de Estados Unidos.

En la Ilustración 1 se grafica la curva de viajes generados por número de empleados am y su relación con la curva del Trip Generation.

Los valores graficados como resultados en Guayaquil corresponden a los conteos de campo y considera como más críticos, 1480 empleados y 1377 viajes. Adicionalmente se incorpora el ajuste al día promedio año que es $7 \%$ menos, esto es 1281 viajes para el mismo número de emplea-

AEROPUERTO COMERCIAL

(021)

Viajes de vehículos vs. Empleados

Estudio: Día de la semana

Hora pico am

Número de estudio:

Distribución direccional:

$53 \%$ entrada; $47 \%$ salida

Generación de Viajes por Empleado

Tasa Promedio Rango de Tasa

0,80

$0,72-0,87$

Desviación Estándar

0,10

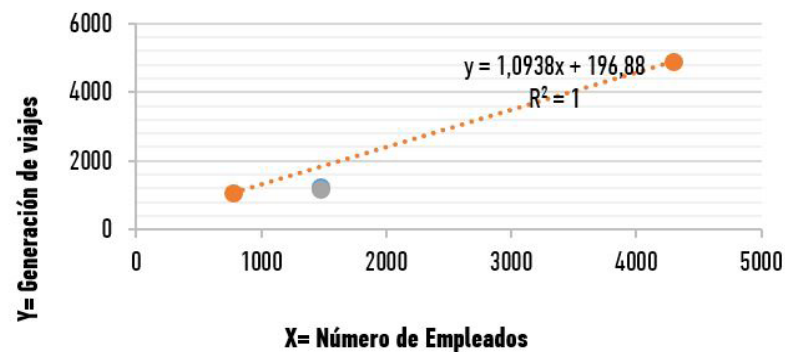

Datos locales

- Trip Generation

- Datos locales ajustados a un

$7 \%$ menos

$X=$ Número de Empleados

Figura 1. Generación de Viajes para el número de empleados, hora pico am. 


\section{AEROPUERTO COMERCIAL}

(021)

\section{Viajes de vehículos vs. Empleados \\ Día de estudio: Día de la semana Hora pico pm}

Número de estudio:

Distribución direccional:

$48 \%$ entrada ; $52 \%$ salida

\section{Generación de Viajes por Empleado}

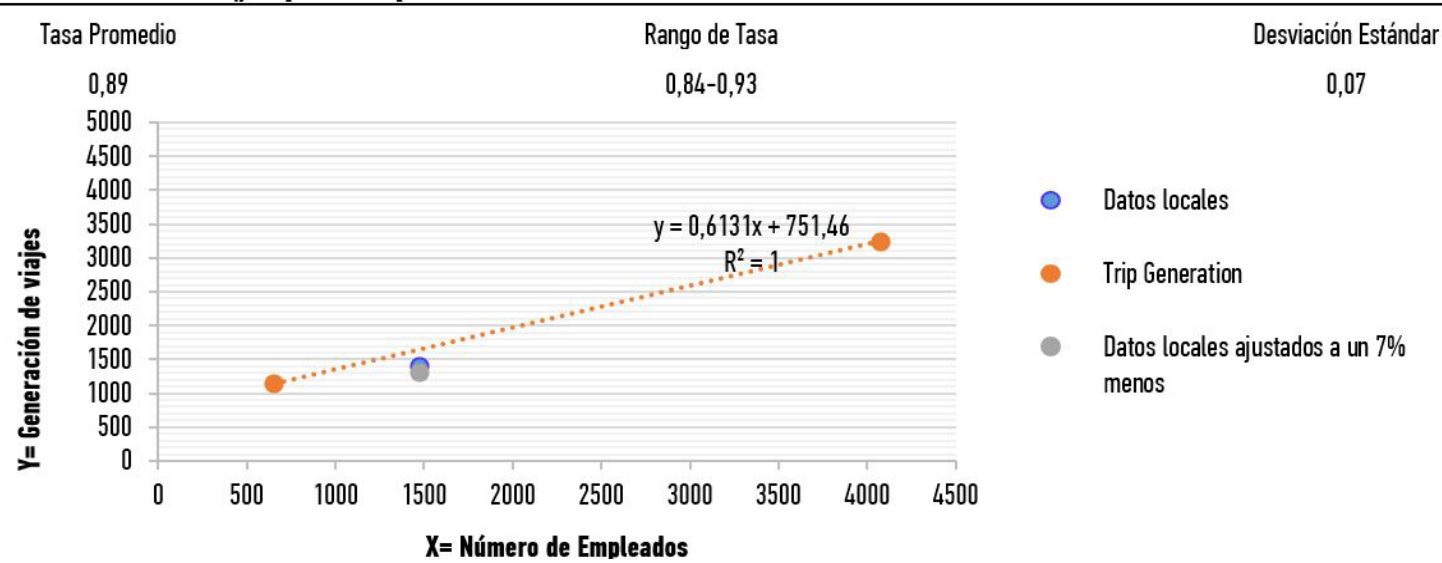

Figura 2. Generación de Viajes para el número de empleados, hora pico pm.

dos (1480). Se aprecia que los resultados de viajes por número de empleados en los EEUU es mayor.

En la Ilustración 2 se grafica la condición de la tarde (pm) y de igual forma se relaciona a los empleados con el número de viajes con la condición más crítica, 1480 empleados y con la reducción del 7\%, (1376 viajes). Con esto se demuestra que la relación número de empleados versus número de viajes, tanto en la mañana como en la tarde en los EE.UU para el mismo número de empleados genera más número de viajes de vehículos.

\section{AEROPUERTO COMERCIAL}

(021)

Viajes de vehículos Vs. Vuelos promedio por día

Estudio:

Día de la semana

Hora pico am

Número de estudio:

Distribución direccional:

$53 \%$ entrada ; $47 \%$ salida

Generación de Viajes por Empleado

$\begin{array}{ccc}\text { Tasa Promedio } & \text { Rango de Tasa } & \text { Desviación Estándar } \\ 10,02 & 9,01-10,85 & 1,30\end{array}$

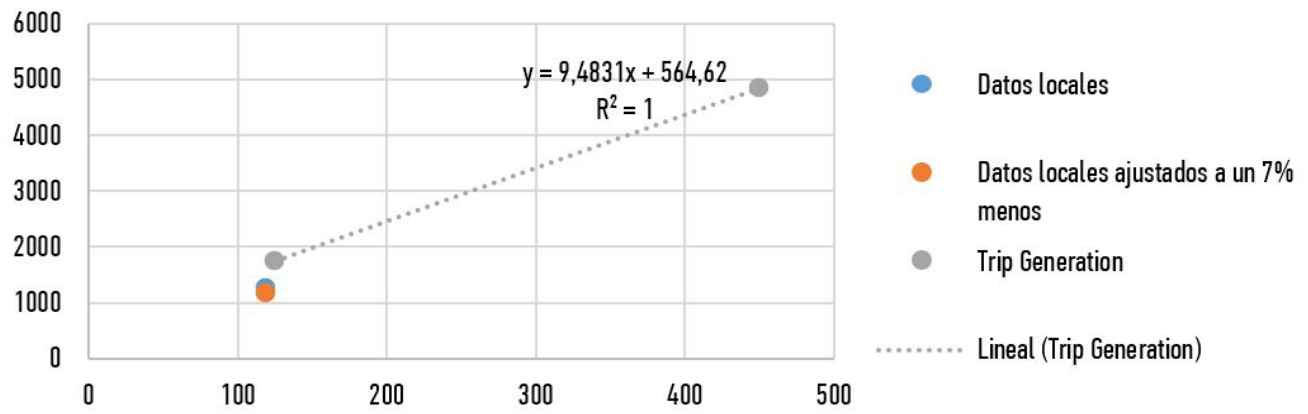

Figura 3. Generación de viajes para el número de vuelos promedio por día, hora pico am. 


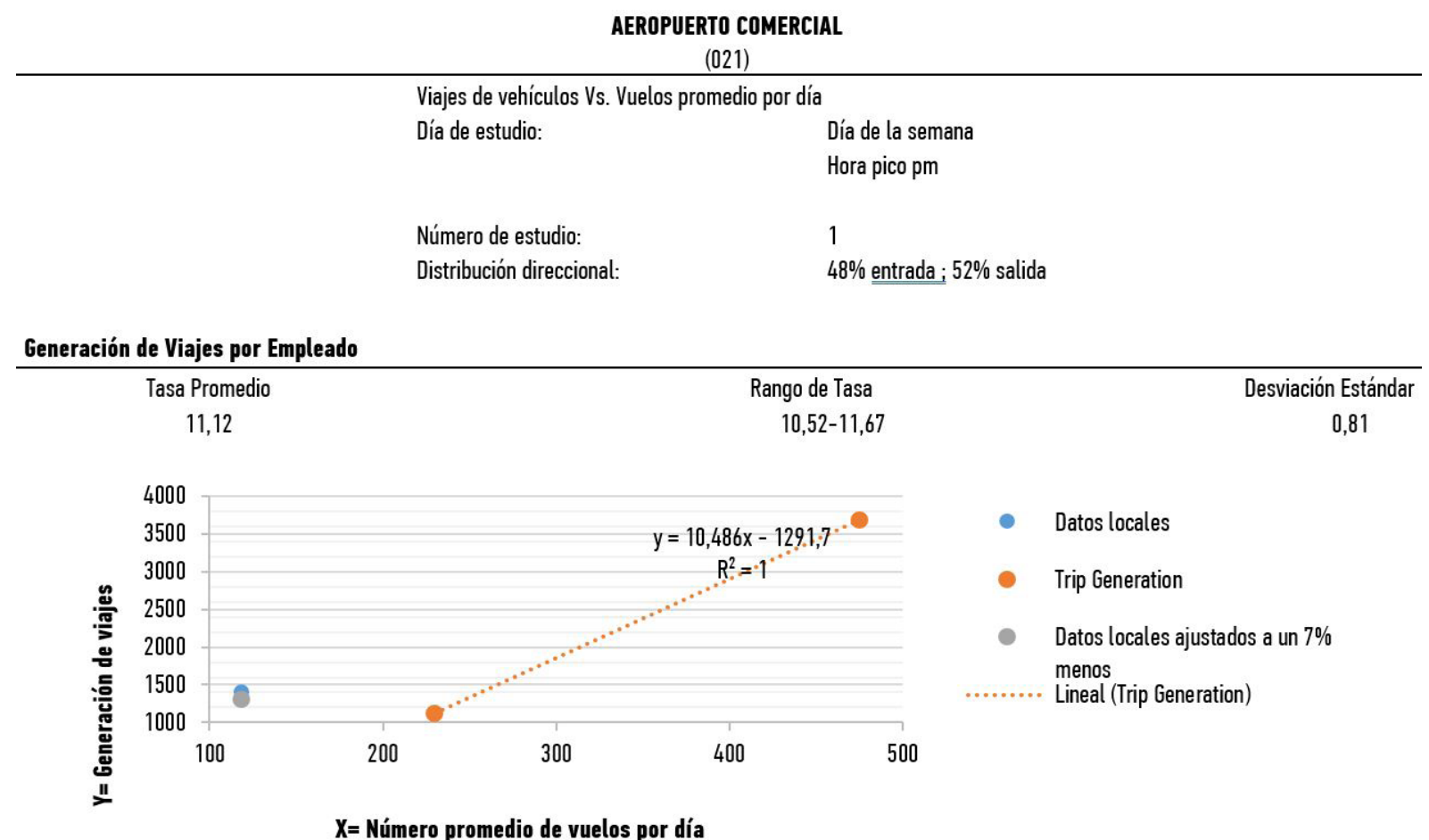

Figura 4. Generación de viajes para el número de vuelos promedio por día, hora pico pm.

La relación de número de viajes promedio por vuelo de aviones por día en la mañana, nos da 1377 vehículos y con la reducción por ajuste al volumen promedio diario nos da 1280 vehículos para 118 vuelos promedio por día (Ver Figura 3). Este valor es inferior a las curvas del Trip Generation Manual.
En la figura 4 se aprecia que los valores mínimos de las curvas del Trip Ceneration Manual son ligeramente mayores al número de vuelos que tiene nuestro aeropuerto y los resultados de su proyección presentan mayor número de viajes.

Al relacionar el número de vuelos comerciales con el número de viajes totales de vehículos se

\section{AEROPUERTO COMERCIAL}

(021)

$\begin{array}{cl}\text { Viajes de vehículos Vs. Vuelos comerciales por día } \\ \text { Día de estudio: } & \text { Día de la semana } \\ & \text { Hora pico am }\end{array}$

Número de estudio: 1

Distribución direccional: $\quad 53 \%$ entrada ; $47 \%$ salida

Generación de Viajes por Empleado

Tasa Promedio Rango de Tasa Desviación Estándar

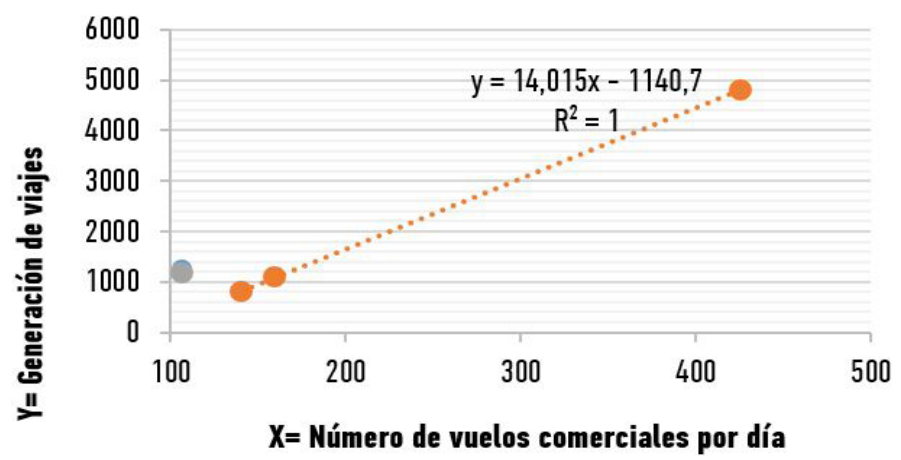

Datos locales

- Trip Generation

- Datos locales ajustados a un $7 \%$ menos Lineal (Trip Generation)

Figura 5. Generación de Viajes para el número de Vuelos comerciales por día, hora pico am. 


\section{AEROPUERTO COMERCIAL}

(021)

\section{Viajes de vehículos Vs. Vuelos comerciales por día}

Día de estudio:

Día de la semana

Hora pico pm

Número de estudio:

Distribución direccional:

$48 \%$ entrada ; $52 \%$ salida

\section{Generación de viajes por empleado}

Tasa Promedio

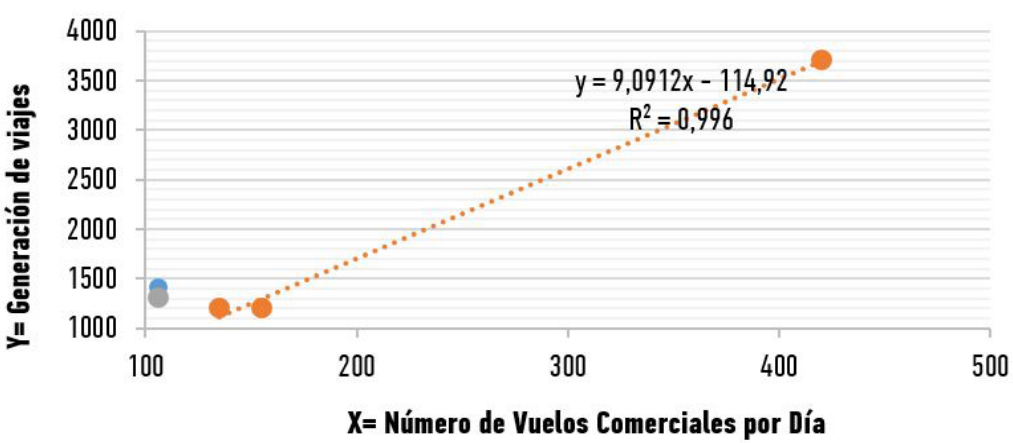

Desviación Estándal

\section{Datos locales}

- Trip Generation

- Datos locales ajustados a un $7 \%$ menos

Figura 6. Generación de viajes para el número de vuelos comerciales por día, hora pico pm.

observa que el máximo que se tiene en la mañana es de 108 viajes y 1377 viajes o 1281 para el valor promedio anual, al comparar con la proyección del Trip Generation Manual daría valores menores (Ver Figura 5). Estos gráficos muestran condiciones diferentes al compararlos con el Trip Generation en la mañana para el mismo número de vuelos promedio por día. Los datos del ITE dan mayor número de viajes, y en la tarde para el mismo número de vuelos el ITE no tiene valores para ese número de vuelos comerciales por día.

La relación de los vuelos comerciales versus el número de viajes de vehículos en la tarde tenemos 108 vuelos comerciales máximo y 1480 viajes considerando el número de viajes promedio día serían 1376 viajes. La proyección de la curva del Trip Generation da valores menores (Ver Figura 6).

CONCLUSIONES

Las tasas de generación de viajes de vehículos por día promedio y por número de empleados oscilan entre 0,7 y 0,9 , considerando los máximos en la mañana y en la tarde, mientras que en los Estados Unidos de Norte América la relación tiene un rango de 1,3 a 1,4. Circunstancia que guarda relación con las de tasas de motoriza- ción de los EEUU que es el $80 \%$ mientras que en Guayaquil es del $14 \%$.

Las relaciones con el número de viajes de vehículos generados por la cantidad de vuelos por día, en la mañana y en la tarde tiene rangos entre 9,8 a 11,7 y la generación de viajes de vehículos por vuelos comerciales tiene rangos entre 9,8 a 12,8, los que al compararlos con las curvas del TCM encontramos que en el único caso en que se dan valores de viajes menores que en los EEUU es en las mañanas, en el resto de las condiciones la relación del número de viajes por número de vuelos promedio y comerciales en Guayaquil son menores que el mínimo que presentan las tablas del Trip Generation Manual, las que al proyectarlas darían menor número de viajes de vehículos que en Guayaquil.

\section{REFERENCIAS BIBLIOGRÁFICAS}

Institute of Transportation Engineers, ITE. (2012). Trip Generation Manual.

ITE. (2004). Trip Generation Handbook 2Nd Ed. Ministerio De Educacion. (2012). Archivo Maestro de Instituciones Educativas-AMIE.

Valarezo, C. (2017). Generación de viajes ajustados a las circunstancias de aeropuerto de la ciudad de Guayaquil. Universidad Católica Santiago de Guayaquil. 